\title{
SOME CHARACTERIZATIONS OF MOUFANG GENERALIZED QUADRANGLES
}

\author{
FABIENNE HAOT and HENDRIK VAN MALDEGHEM \\ Ghent University, Department of Pure Mathematics and Computer Algebra, \\ Galglaan 2, 9000 Gent, Belgium \\ e-mail:fhaot@cage.rug.ac.be,hvm@cage.rug.ac.be
}

(Received 5 May, 2003; accepted 9 October 2003)

\begin{abstract}
We prove the longstanding conjecture that the 3-Moufang condition for generalized quadrangles is equivalent to the Moufang condition. We mention some other characterizations of Moufang quadrangles that follow from this result. We also provide a short proof of Tent's recent result that every half Moufang quadrangle is necessarily a Moufang quadrangle.
\end{abstract}

2000 Mathematics Subject Classification. 51E12.

1. Introduction. Generalized quadrangles were introduced by Tits [12] as the natural incidence geometries related to the classical, algebraic and mixed groups of relative type $\mathrm{B}_{2}$. If we call these the natural examples, then Tits [13] conjectured that the only generalized quadrangles satisfying the so-called Moufang condition (see below for precise definitions) are the natural examples. In the finite case, this conjecture was known to be true by group-theoretic work of Fong and Seitz [5, 6]. A lot of alternative conditions have been established in the finite case to characterize the natural examples. Almost all of them were proved by reducing the conditions to the Moufang condition. This was done by applying some typical finiteness arguments (for instance, counting and finite group theory).

In the general case, the classification of all Moufang quadrangles was technically only finished in 1997, when a new and final class was discovered. The classification and its proof recently appeared in the monograph [16] by Tits and Weiss. Hence people want to know whether the characterization theorems of the finite case can be extended to the general case. There are four main global characterizations of all finite Moufang quadrangles that can be considered here. We quickly review the conditions.

1. The automorphism group of the generalized quadrangle acts flag-transitively, the stabilizer $B$ of a flag $F$ acts transitively on the ordinary quadrangles containing $F$, and it contains a transitive normal nilpotent subgroup $U$.

Such groups are usually called groups with a split BN-pair of type $\mathrm{B}_{2}$. In the finite case, they were classified by Fong and Seitz $[\mathbf{5}, \mathbf{6}]$. In the general case, Tent and Van Maldeghem [8] show that this condition implies the Moufang condition. The converse was already known to be true, see Tits [15].

2. The half Moufang condition.

In the finite case, Thas, Payne and Van Maldeghem [10] showed that this condition implies the Moufang condition. In the general case, Tent constructed a proof of this fact using a certain lemma. This lemma turned out to be wrong when Weiss found a counterexample (private communication; we will refer to that lemma below as the 
"wrong lemma"). However, the second author could repair Tent's proof using an alternative argument (and this is contained in [7]). In fact, all that is needed to prove the general case is that argument and one observation by Tent. We show how this can be done at the end of the paper.

3. The $k$-Moufang condition, $k \in\{2,3\}$.

In the finite case, Van Maldeghem, Thas and Payne [18] showed that 3-Moufang implies Moufang (for generalized quadrangles). Later on, the second author of the present paper [17] showed that the 2-Moufang condition is equivalent with the 3Moufang condition for all generalized quadrangles. In the present paper, we will show that the 3-Moufang condition implies the Moufang condition for (not necessarily finite) generalized quadrangles.

For $k=2$, there is also the notion of a half 2-Moufang condition. In the finite case, it has recently been proved by Thas and Van Maldeghem [11] that finite half 2-Moufang quadrangles are automatically Moufang. In the infinite case, this is still open. The proof in the finite case again relies heavily on finiteness techniques.

4. The automorphism group of the generalized quadrangle acts distance-transitively on the point set of the quadrangle.

In the finite case, this condition implies the Moufang condition, as has been showed by Buekenhout and Van Maldeghem [2] using the classification of finite simple groups, in particular the classification of primitive rank 3 groups. This result implies all previous characterizations in the finite case. There is no hope for a proof in the general case as there are free constructions of quadrangles with an automorphism group acting transitively on ordered ordinary quadrangles starting with a point, see [14] (cp. also $[\mathbf{1 7}, 4.7 .1])$. In fact, the proof in the finite case does not give any information or insight.

As mentioned above, in this paper we will show that the 3-Moufang condition implies the Moufang condition for an arbitrary generalized quadrangle. This problem was open since 1992. We will also mention some corollaries, and give a short proof of the implication half Moufang to Moufang.

Finally, to conclude this introduction, we mention that all notions can be put in the general framework of generalized polygons. The situation there is, however, not so satisfying as in the case of quadrangles, although in the finite case most characterizations can be generalized from quadrangles to polygons. In the infinite case there are only some sporadic and partial results.

2. Definitions, notation and results. A generalized quadrangle of order $(s, t), s, t \in$ $\mathbf{N} \cup\{\infty\}$ is an incidence structure $\mathcal{S}=(\mathcal{P}, \mathcal{L}, I)$ in which $\mathcal{P}$ and $\mathcal{B}$ are disjoint (nonempty) sets of objects called points and lines respectively, and for which $I$ is a symmetric point-line incidence relation satisfying the following properties.

(i) Each point is incident with $t+1$ lines $(t \geq 1)$ and two distinct points are incident with at most one line.

(ii) Each line is incident with $s+1$ points $(s \geq 1)$ and two distinct lines are incident with at most one point.

(iii) If $(x, L)$ is a non-incident point-line pair then there is a unique point-line pair $(y, M)$ for which $x I M I y I L$.

If $s, t \geq 2$ we call our geometry thick. Non-thick generalized quadrangles are rather trivial geometries, called grids and dual grids. From now on, we only consider thick generalized quadrangles.

We first remark that for generalized quadrangles we have the principle of duality. That means that, if we interchange the roles of the point set and the line set in a 
theorem, we obtain another theorem (which may or may not be different from the original theorem).

We will use the following terminology and notation. Let $\mathcal{S}=(\mathcal{P}, \mathcal{L}, I)$ be a thick generalized quadrangle. In Condition (iii) the point $y$ and the line $M$ are called the projection of $x$ onto $L$ and of $L$ onto $x$, respectively, and denoted by $\operatorname{proj}_{L} x$ and $\operatorname{proj}_{x} L$, respectively. Two points $x, y$ incident with a common line are collinear, and two lines $L, M$ incident with a common point are concurrent. We write this as $x \perp y$ and $L \perp M$. If $x \neq y$, then the line incident with them is unique and denoted by $x y$; similarly if $L \neq M$, then $L \cap M$ is the unique point incident with both. Two points or two lines which are not collinear or concurrent, respectively, are called opposite. If $S$ is a set of points or lines, then $S^{\perp}$ denotes the set of points or lines collinear or concurrent with every point or line of $S$, respectively. A $k$-path, $k \geq 0$, is a sequence $\left(x_{0}, x_{1}, \ldots, x_{k}\right)$ of points and lines of $\mathcal{S}$ such that $x_{i-1} I x_{i}, 1 \leq i \leq k$, and such that $x_{i-1} \neq x_{i+1}, 1 \leq i<k$. An incident point-line pair is a flag. A subquadrangle $\mathcal{S}^{\prime}$ of $\mathcal{S}$ consists of a point set $\mathcal{P}^{\prime} \subseteq \mathcal{P}$ and a line set $\mathcal{L}^{\prime} \subseteq \mathcal{L}$ such that, if we denote by $I^{\prime}$ the restriction of $I$ to $\mathcal{P}^{\prime} \cup \mathcal{L}^{\prime}$, the structure $\mathcal{S}^{\prime}=\left(\mathcal{P}^{\prime}, \mathcal{L}^{\prime}, I^{\prime}\right)$ is a generalized quadrangle. The subquadrangle $\mathcal{S}^{\prime}$ is called full (ideal) if for every line $L^{\prime} \in \mathcal{L}^{\prime}$ (point $x^{\prime} \in \mathcal{P}^{\prime}$ ) all points (lines) of $\mathcal{S}$ incident with $L^{\prime}\left(x^{\prime}\right)$ belong to $\mathcal{S}^{\prime}$. An apartment is a set of 4 points and 4 lines which form a subquadrangle of order $(1,1)$.

A collineation of $\mathcal{S}$ is a permutation of $\mathcal{P} \cup \mathcal{L}$ inducing permutations of $\mathcal{P}$ and $\mathcal{L}$, and preserving incidence and non-incidence. We denote by $G$ a collineation group of $\mathcal{S}$. With regard to permutation groups, we always use right action and exponential notation (so the image of an element $x$ under the collineation $u$ is written as $x^{u}$ ). The commutator $[u, v]$ of two collineations is $u^{-1} v^{-1} u v$ and acts on $x$ as $x^{[u, v]}=$ $\left(\left(\left(x^{u^{-1}}\right)^{v^{-1}}\right)^{u}\right)^{v}$. The conjugate $u^{v}$ is equal to $v^{-1} u v$. We will always denote the identity by id, and for a group $H$, we denote $H^{\times}=H \backslash\{\mathrm{id}\}$.

Consider a $(k-2)$-path $C=\left(x_{1}, \ldots, x_{k-1}\right)$, with $2 \leq k \leq n$, in the generalized quadrangle $\mathcal{S}$ and let $x_{0}, x_{k} \in \mathcal{P} \cup \mathcal{L}$ be such that $\bar{C}=\left(x_{0}, x_{1}, \ldots, x_{k-1}, x_{k}\right)$ is a $k$ path. If the group $G^{\left[x_{1}, \ldots, x_{k-1}\right]}$ of collineations fixing every element incident with an element of $C$ acts transitively on the set of apartments containing the $k$-path $\bar{C}$, then $C$ is called a Moufang path (with respect to $G$ ). We easily check that this is independent of the choice of $x_{0}$ and $x_{k}$. If every $(k-2)$-path is a Moufang path, then $\mathcal{S}$ is called a $k$-Moufang quadrangle (with respect to $G$ ). Note that it is well known that, for $k \in\{3,4\}$, the group $G^{\left[x_{1}, \ldots, x_{k-1}\right]}$ generally acts semi-regularly on the set of apartments containing $\bar{C}$ (see for instance [17, 4.4.2]). A 4-Moufang quadrangle is also called a Moufang quadrangle.

For $k \in\{2,4\}$, there are 2 kinds of $(k-2)$-paths: those containing fewer lines than points, and those containing fewer points than lines. If all $(k-2)$-paths of one type are Moufang paths, then we call $\mathcal{S}$ half $k$-Moufang. A half 4-Moufang quadrangle is also called a half Moufang quadrangle.

We can now state our main result.

MAIN RESUlT. Every 3-Moufang generalized quadrangle with respect to some collineation group $G$ is a Moufang generalized quadrangle with respect to $G$.

We mention a few consequences.

COROLLARY 1. Every 2-Moufang generalized quadrangle with respect to some collineation group $G$ is a Moufang generalized quadrangle with respect to $G$. 
An elation point $x$ of a generalized quadrangle $\mathcal{S}$ is a point such that $G^{[x]}$ contains some subgroup $H$ acting regularly on the set of points opposite $x$, where this time, $G$ is the full collineation group. Dually, one defines an elation line.

COROLlary 2. A generalized quadrangle is a Moufang quadrangle if and only if it has at least two opposite elation points and two opposite elation lines.

Let $x, y$ be two opposite points of the generalized quadrangle $\mathcal{S}$. Then we say that $\mathcal{S}$ is $\{x, y\}$-transitive if, for some (and hence for every) line $L$ incident with $x$ the collineation group $G^{[x]} \cap G^{[y]}$ (where again $G$ is the full collineation group) acts transitively on the set of points incident with $L$, but distinct from $x$ and $\operatorname{proj}_{L} y$. It is easy to see that this definition is symmetric in $x$ and $y$. Dually, one defines $\{L, M\}$ transitivity, for opposite lines $L, M$.

COROLLARY 3. If a generalized quadrangle is $\{x, y\}$-transitive for all pairs of opposite points $x, y$, and $\{L, M\}$-transitive for all pairs of opposite lines $L, M$, then it is a Moufang quadrangle.

We remark that not every Moufang quadrangle contains opposite points or lines $a, b$ such that it is $\{a, b\}$-transitive. But it is not so hard to deduce from the classification of Moufang quadrangles and the information on their collineation groups provided in [16] that exactly the ones whose "root groups" are all parametrized by skew fields satisfy the hypotheses of the above corollary (the "root groups" are the groups $G^{\left[x_{1}, x_{2}, x_{3}\right]}$, for 2-paths $\left.\left(x_{1}, x_{2}, x_{3}\right)\right)$.

In the finite case, Thas [9] showed that $\{x, y\}$-transitivity for all pairs of opposite points $x, y$ implies the Moufang condition (and, up to duality, every finite Moufang quadrangle is $\{x, y\}$-transitive, for all pairs of opposite points $x, y$ ). The above corollary is not yet as strong as this finite analogue, but it is the first of its kind valid for infinite generalized polygons.

In the next Section we prove our main result by showing that every 3-Moufang quadrangle is a half Moufang quadrangle. In Section 4 we prove the corollaries, and in Section 5 we provide a short proof of the fact that half Moufang quadrangles are Moufang quadrangles.

3. Proof of the main result. In this section, we denote by $\mathcal{S}=(\mathcal{P}, \mathcal{L}, I)$ a thick generalized quadrangle satisfying the 3-Moufang condition. We choose an arbitrary apartment $\Sigma$ and put $\Sigma=\left\{x_{0}, X_{1}, x_{2}, \ldots, x_{6}, X_{7}\right\}$, where we read the subscripts modulo 8 , with $x_{2 i} I X_{2 i \pm 1}$, for all $i \in \mathbb{Z} \bmod 8$. As the notation suggests, we view the $x_{2 i}$ as points and the $X_{2 i+1}$ as lines.

LEMMA 1. The group $G$ acts transitively on each type of 2-paths in $\mathcal{S}$.

Proof. Since the 3-Moufang condition is self-dual, we may restrict to 2-paths containing two points and one line. Let $x_{6}^{\prime}$ be an arbitrary point collinear with $x_{0}$. Since $\mathcal{S}$ is thick, we may assume without loss of generality that $x_{6}^{\prime}$ is not incident with $X_{1}$. Hence, there is a unique apartment $\Sigma^{\prime}$ containing the points $x_{6}^{\prime}, x_{0}, x_{2}$ and $\operatorname{proj}_{X_{3}} x_{6}^{\prime}$. By the 3-Moufang condition, there is a collineation $u \in G^{\left[x_{1}, x_{2}\right]}$ mapping $\Sigma$ to $\Sigma^{\prime}$, and hence $x_{6}$ to $x_{6}^{\prime}$. First of all, this implies that $G$ is transitive on the point set $\mathcal{P}$ of $\mathcal{S}$; secondly, this shows that the stabilizer of the point $x_{0}$ acts transitively on the 2-paths containing $x_{0}$ and some further point. The lemma is proved. 
In fact, it is not hard to see that $G$ acts transitively on each type of 4-paths of $\mathcal{S}$. This implies that everything we prove or assume for one 2- or 4-path automatically holds for every 2- or 4-path of the same type. For instance, if $G_{X_{5}}^{\left[x_{2}, X_{3}\right]}$ is abelian, then $G_{X_{2 i+3}}^{\left[x_{2 i}, X_{2 i+1}\right]}$ is abelian for all $i$ modulo 8 . We will use things like that freely in the sequel.

LEMMA 2. Let $u \in G_{X_{5}}^{\left[x_{2}, X_{3}\right]}$ and suppose that $u$ fixes some line $X_{5}^{\prime}$ concurrent with $X_{3}$, but not incident with $x_{2}$ or with $x_{4}$. Then $u \in G^{\left[x_{4}\right]}$. Hence, by symmetry, if $x_{4}^{\prime}=X_{5}^{\prime} \cap X_{3}$, then also $u \in G^{\left[x_{4}^{\prime}\right]}$.

Proof. Let $w \in G^{\left[x_{4}, X_{5}\right]}$ be such that it maps $X_{1}$ to $X_{5}^{\prime}$ (and so it maps $x_{2}$ to $x_{4}^{\prime}$ ). Then $\left[u^{-1}, w\right]$ belongs to $G^{\left[X_{3}, x_{4}, X_{5}\right]}$ and fixes additionally $X_{1}$. Hence $\left[u^{-1}, w\right]=$ id. So $u=u^{w}$. But $u^{w} \in\left(G^{\left[x_{2}, X_{3}\right]}\right)^{w}=G^{\left[x_{2}^{w}, X_{3}^{w}\right]}=G^{\left[x_{4}^{\prime}, X_{3}\right]}$, hence $u$ fixes all lines through $x_{4}^{\prime}$. Similarly, $u$ also fixes all lines through $x_{4}$. The lemma is proved.

LemMA 3. Suppose that $U_{3}:=G_{X_{5}}^{\left[x_{2}, X_{3}\right]}$ is nonabelian. Then there exists a nontrivial element $u \in G_{X_{5}}^{\left[x_{2}, X_{3}\right]}$ fixing all lines concurrent with $X_{3}$.

Proof. Let $u_{3}, u_{3}^{\prime} \in U_{3}$ be arbitrary but such that $u=\left[u_{3}, u_{3}^{\prime}\right]$ is nontrivial. This is possible by our assumption. Choose an arbitrary point $x_{2}^{\prime} \neq x_{4}$ incident with $X_{3}$. Let $w \in G^{\left[x_{4}, X_{5}\right]}$ be such that it maps $x_{2}$ to $x_{2}^{\prime}$. Then $u_{3}^{w}$ has the same action on the set of points incident with $X_{5}$ as $u_{3}$. Hence $\left[u_{3}^{w}, u_{3}^{\prime}\right]$ has the same action on the set of points incident with $X_{5}$ as $\left[u_{3}, u_{3}^{\prime}\right]$. Moreover, $\left[u_{3}^{w}, u_{3}^{\prime}\right] \in G_{x_{5}}^{\left[x_{2}, X_{3}\right]} \cap G_{X_{5}}^{\left[x_{2}^{\prime}, X_{3}\right]}$. So, using the semi-regularity of the action of $G^{\left[x_{2}, X_{3}\right]}$ on the set of apartments containing $X_{1}$ and $x_{4}$, we deduce that $u=\left[u_{3}^{w}, u_{3}^{\prime}\right] \in G^{\left[x_{2}^{\prime}\right]}$. We conclude that, since $x_{2}^{\prime}$ was arbitrary, $u$ fixes all lines concurrent with $X_{3}$, except possibly those incident with $x_{4}$. But Lemma 2 now guarantees that also all these lines are fixed. The lemma is proved.

LEMMA 4. If $\left\{X_{3}, X_{7}\right\}^{\perp \perp}$ contains at least three elements, then $\mathcal{S}$ is half Moufang.

Proof. Recall that $\left\{X_{3}, X_{7}\right\}^{\perp \perp}$ is the set of all lines concurrent with every line that is concurrent with both $X_{3}$ and $X_{7}$. Since by assumption there are at least three such lines, we may choose one, say $X_{7}^{\prime}$, which is different from both $X_{3}$ and $X_{7}$. Let $u \in G_{X_{3}}^{\left[x_{0}, X_{1}\right]}$ be arbitrary. Since $u$ fixes $X_{3}$ and $X_{7}$, it stabilizes $\left\{X_{3}, X_{7}\right\}^{\perp \perp}$ setwise. But $X_{7}^{\prime}$ is the unique element of $\left\{X_{3}, X_{7}\right\}^{\perp \perp}$ incident with the point $X_{7}^{\prime} \cap X_{1}$, which is fixed by $u$. Hence $u$ fixes $X_{7}^{\prime}$ and Lemma 2 implies $u \in G^{\left[x_{0}, X_{1}, x_{2}\right]}$, from which we conclude that $G^{\left[x_{0}, X_{1}, x_{2}\right]}$ acts transitively on the set of apartments containing $X_{7}, X_{1}$ and $X_{3}$. An application of Lemma 1 completes the proof.

We can now finish the case where $U_{3}:=G_{X_{5}}^{\left[x_{2}, X_{3}\right]}$ is nonabelian. Indeed, let $u \in$ $G_{X_{5}}^{\left[x_{2}, X_{3}\right]}$ be nontrivial and such that it fixes all lines concurrent with $X_{3}$ (see Lemma 3 ). Then $X_{7}^{\prime}:=X_{7}^{u}$ is concurrent with every line that meets both $X_{3}$ and $X_{7}$, and hence belongs to $\left\{X_{3}, X_{7}\right\}^{\perp \perp}$. Lemma 4 implies that $\mathcal{S}$ is half Moufang, and hence a Moufang quadrangle by [7] (see also Section 5).

From now on we may assume that the groups $G_{X_{5}}^{\left[x_{2}, X_{3}\right]}$ and $G_{x_{4}}^{\left[X_{1}, x_{2}\right]}$ are abelian. Note that these groups act sharply transitively on the elements of $\mathcal{S}$ incident with $X_{5}$ and $x_{4}$, respectively, distinct from $x_{4}$ and $X_{3}$, respectively. Also, it is clear that $\left[G_{X_{5}}^{\left[x_{2}, X_{3}\right]}, G_{X_{1}}^{\left[x_{4}, X_{3}\right]}\right] \leq G_{X_{5}}^{\left[x_{2}, X_{3}\right]} \cap G_{X_{1}}^{\left[x_{4}, X_{3}\right]}$, hence $G_{X_{5}}^{\left[x_{2}, X_{3}\right]}$ and $G_{X_{1}}^{\left[x_{4}, X_{3}\right]}$ normalize each other. About regular abelian groups normalizing each other, there is the following result (which is weak version of the "wrong lemma" mentioned in the introduction). The proof is an easy exercise, but we provide one for completeness' sake. 
Lemma 5. Let $(H, \Omega)$ be a permutation representation and let $H_{i} \leq H, i=1,2$, be two abelian subgroups acting regularly on $\Omega$. Suppose $\left[H_{1}, H_{2}\right] \leq H_{1} \cap H_{2}$ (in other words, $H_{1}$ and $H_{2}$ normalize each other). Let $x \in \Omega$ be arbitrary, and suppose that $h_{1} \in H_{1}$ and $h_{2} \in H_{2}$ are such that $x^{h_{1} h_{2}}=x$. Then $\left|x^{H_{1} \cap H_{2}}\right|>1$ and $y^{h_{1} h_{2}}=y$, for all $y \in x^{H_{1} \cap H_{2}}$.

Proof. It is well known that, if $\left[H_{1}, H_{2}\right]$ is trivial, then $H_{1}=H_{2}$ (as permutation groups acting on $X$ ), see for instance [4]. In this case, clearly $y^{h_{1} h_{2}}=y$, for all $y \in \Omega$, since $h_{1}=h_{2}^{-1}$. Hence we may assume that $\left[H_{1}, H_{2}\right]$ is nontrivial, and hence $H_{1} \cap H_{2}$ is nontrivial.

Pick $y \in x^{H_{1} \cap H_{2}}$ arbitrary, and let $h_{1}$ and $h_{2}$ be as in the statement of the lemma. Let $h \in H_{1} \cap H_{2}$ be such that $x^{h}=y$. Then $y^{h_{1} h_{2}}=x^{h h_{1} h_{2}}=x^{h_{1} h_{2} h}=x^{h}=y$ (since $h$ centralizes both $H_{1}$ and $H_{2}$ ).

The lemma is proved.

We now complete the proof of our main result.

We may assume that $\mathcal{S}$ is not a half Moufang generalized quadrangle, hence, without loss of generality, there exists a nontrivial element $u \in G_{X_{5}}^{\left[x_{2}, X_{3}\right]} \backslash G_{X_{1}}^{\left[x_{4}, X_{3}\right]}$. Let $\Omega$ be the set of all apartments containing $X_{1}, X_{3}$ and $X_{5}$. Note that $\Sigma \in \Omega$. By the foregoing lemma there are an element $u^{\prime} \in G_{X_{1}}^{\left[x_{4}, X_{3}\right]} \backslash G_{X_{5}}^{\left[x_{2}, X_{3}\right]}$, and a subset $\Omega^{\prime} \subseteq \Omega$ containing $\Sigma$, with $\left|\Omega^{\prime}\right|>1$, such that $u^{-1} u^{\prime}$ fixes all elements of $\Omega^{\prime}$.

If $\Omega^{\prime} \neq \Omega$, then $u^{-1} u^{\prime}$ fixes $\left\{X_{3}, X_{7}\right\}^{\perp}$ and at least three, but not all elements of $\left\{X_{5}, X_{1}\right\}^{\perp}$. We claim that all lines of $\left\{X_{5}, X_{1}\right\}^{\perp}$ that are fixed under $u^{-1} u^{\prime}$ belong to $\left\{X_{3}, X_{7}\right\}^{\perp \perp}$. Indeed, if not, then let us consider a line $L \in\left\{X_{5}, X_{1}\right\}^{\perp}$ fixed under $u^{-1} u^{\prime}$, but not belonging to $\left\{X_{3}, X_{7}\right\}^{\perp \perp}$. Put $L \cap X_{1}=x_{0}^{\prime}$. Hence there is a line $Q$ in $\left\{X_{3}, X_{7}\right\}^{\perp}$ which is not concurrent with $L$. Thus $L$ and $Q$ are opposite, so $x_{0}^{\prime}$ is not incident with $Q$. Since $u^{-1} u^{\prime}$ fixes all elements of $\left\{X_{3}, X_{7}\right\}^{\perp}$, it fixes $Q$. Therefore $u^{-1} u^{\prime}$ fixes the unique 3 -path from $x_{0}^{\prime}$ to $Q$. Consequently, $u^{-1} u^{\prime}$ fixes at least three lines incident with $x_{0}^{\prime}$. This implies that it fixes a thick full subquadrangle, implying (by [17, Proposition 1.8.1]) that all points incident with $X_{1}$ are fixed. This contradicts our assumption $\Omega^{\prime} \neq \Omega$. The claim is proved. But now Lemma 4 shows that $\mathcal{S}$ is a half Moufang quadrangle.

Hence we may assume that $u^{-1} u^{\prime}$ fixes all lines $X_{1}, X_{3}, X_{5}, X_{7}$ pointwise, but does not act trivially on the set of lines incident with $x_{2}$. It is more convenient to argue with the dual situation. Dually, we have a collineation $w$ fixing all lines incident with the points $x_{0}, x_{2}, x_{4}, x_{6}$, but not fixing all points of the line $X_{1}$. In fact, we may re-name the points incident with $X_{1}$ in such a way that $w$ maps $x_{0}$ onto $x_{2}$, and $w$ fixes all lines incident with some points $x_{2}^{\prime}$ and $x_{0}^{\prime}$, with $x_{2}^{\prime} I X_{1} I x_{0}^{\prime}$. We now take an arbitrary element $v \in G_{X_{3}}^{\left[x_{0}, X_{1}\right]}$. Since we assume that $\mathcal{S}$ is not half Moufang, we can choose $v$ such that it does not fix all lines incident with $x_{2}$. Now consider the commutator $[v, w]$. It certainly fixes all lines incident with $x_{2}^{\prime}$ and those incident with $x_{0}^{\prime}$, and it fixes all points incident with $X_{1}$. Now, $v^{-1}$ does not fix all lines incident with $x_{2}$. Also, $w^{-1}$ maps the lines incident with $x_{2}$ onto the lines incident with $x_{0}$. Finally, $v$ fixes all these lines. So we see that $[v, w]$ does not fix all lines incident with $x_{2}$. Lemma 2 implies that $[v, w]$ cannot fix any line incident with $x_{2}$. But this contradicts $X_{3}^{[v, w]}=X_{3}^{w^{-1} v w}=\left(\left(X_{3}^{w^{-1}}\right)^{v}\right)^{w}=X_{3}^{w^{-1} w}=X_{3}$.

The proof of the Main Result is complete.

4. Proof of the corollaries. Corollary 1 follows directly from the fact that 2-Moufang quadrangles are automatically 3-Moufang quadrangles (see [17]). 
Concerning Corollary 2, we first remark that any elation point $x$ defines a Moufang 0 -path $(x)$. If $x, y$ are two opposite elation points, then all points that are opposite $x$ or $y$ are elation points (by transitivity). It is now easy to see that every point is an elation point. Similarly every line is an elation line. Consequently every 0-path is a Moufang 0 -path and the quadrangle is 2-Moufang.

Now suppose $\mathcal{S}$ satisfies the assumptions of Corollary 3. Let $L$ be any line of $\mathcal{S}$. Let $M$ and $M^{\prime}$ be two lines opposite $L$. If we show that there is a collineation $u \in G^{[L]}$ mapping $M$ to $M^{\prime}$, then $(L)$ is a Moufang 0-path and, together with the dual argument, $\mathcal{S}$ is 2-Moufang, proving the corollary.

If $M$ is different from, concurrent with $M^{\prime}$, then let $x$ be the unique point incident with both $M$ and $M^{\prime}$. Remark that we may assume that there are at least 4 lines incident with a given point by [9] and [3]. Hence there is some line $N \notin\left\{M, M^{\prime}, \operatorname{proj}_{x} L\right\}$ incident with $x$. The $\{L, N\}$-transitivity implies that there is some collineation in $G^{[L]}$ mapping $M$ to $M^{\prime}$. If $M$ is not concurrent with $M^{\prime}$, then by a result of Cuypers stated in [1] (see also $[\mathbf{1 7}, 1.7 .15])$, there is a sequence of $\ell$ lines $\left(M=M_{1}, M_{2}, \ldots, M_{\ell}=M^{\prime}\right)$ such that $M_{i} \perp M_{i+1}$, for all $i \in\{1,2, \ldots, \ell-1\}$, and all $M_{i}$ are opposite $L$. The result is now clear.

5. Half Moufang quadrangles. In this section, we show that half Moufang quadrangles are always Moufang quadrangles. The proof we present differs from the one in [7] in that we almost exclusively use the argument of the second author that replaced the "wrong lemma" in [7]. We will indicate the place where we actually borrow an argument of [7].

First, let us mention the following well known result [8]. We keep the notation of Section 3 regarding the apartment $\Sigma$ and its elements.

LEMMA 6. Let $\mathcal{S}$ be a half Moufang quadrangle and suppose all 2-paths containing two lines satisfy the Moufang condition. Then $\mathcal{S}$ is a Moufang quadrangle whenever the following condition is satisfied. Let $x \neq x_{0}$ be arbitrary on the line $X_{1}$, and let $Y \neq X_{1}$ be arbitrary through $x$. Then the action of the collineation group $G^{\left[X_{1}, x, Y\right]}$ on the set of lines incident with $x_{0}$ does not depend on the choice of $x, Y$.

Furthermore, we know from elementary group theory that, if $K$ and $K^{\prime}$ are two groups acting regularly on a set $\Omega$, and if $K$ and $K^{\prime}$ centralize each other, then, without loss of generality, we may identify $\Omega$ with $K$ such that the action of $K$ on $\Omega=K$ is given by right multiplication. Moreover, $K^{\prime} \cong K$, with the action of $h^{\prime} \in K^{\prime}$ on $\Omega=K$ given by left multiplication with $h^{-1}$. We will denote that action of $K^{\prime}$ on $\Omega$ by $K^{\text {opp }}$. We will also use this notation if both $K$ and $K^{\prime}$ fix an element $\omega \in \Omega$ and act regularly on $\Omega \backslash\{\omega\}$.

We assume that $\mathcal{S}$ is a half Moufang quadrangle and prove it is a Moufang quadrangle. We use the same notation as in Section 3, and as in Lemma 6 above (as it suffices to show that the conditions of that lemma are satisfied).

So we have to show that the action of $G^{\left[X_{1}, x, Y\right]}$ on the lines incident with $x_{0}$ does not depend on $x, Y$. This is clear if $x=x_{2}$, because the half Moufang conditions implies the existence of a collineation fixing all lines incident with $x_{0}$ and mapping $X_{3}$ to $Y$ in this case. So we may assume $x \neq x_{2}$, and $Y$ concurrent with $X_{5}$. Put $U_{2}:=G^{\left[X_{1}, x_{2}, X_{3}\right]}$ and $U_{2}^{\prime}:=G^{\left[X_{1}, x, Y\right]}$. Let $\Omega$ be the set of all lines of $\mathcal{S}$ incident with $x_{0}$. For a subgroup $H$ of the stabilizer of $x_{0}$ in $\mathcal{S}$, we denote by $H_{\Omega}$ the permutation group induced by $H$ on 
$\Omega$. We first show that $\left(U_{2}\right)_{\Omega}$ and $\left(U_{2}^{\prime}\right)_{\Omega}$ normalize each other. We borrow the argument from [7]. Let $u_{2} \in U_{2}$ and $u_{2}^{\prime} \in U_{2}^{\prime}$ be arbitrary, and let $u_{8} \in G^{\left[X_{7}, x_{0}, X_{1}\right]}$ be such that $X_{3}^{u_{2}^{\prime} u_{8}}=X_{3}$. Clearly $\left[u_{2}^{\prime} u_{8}, u_{2}\right] \in U_{2}$. But the action of $\left[u_{2}^{\prime} u_{8}, u_{2}\right]$ on $\Omega$ coincides clearly with the action of $\left[u_{2}^{\prime}, u_{2}\right]$ on $\Omega$, since $u_{8}$ acts trivially on $\Omega$. Similarly, the action of $\left[u_{2}^{\prime}, u_{2}\right]$ on $\Omega$ is the same as that of some element of $U_{2}^{\prime}$ on $\Omega$. Hence the actions of $U_{2}$ and $U_{2}^{\prime}$ on the set $\Omega$ normalize each other.

Put $U_{6}:=G^{\left[X_{5}, x_{6}, X_{7}\right]}$. The group $H:=\left\langle U_{6}, U_{2}\right\rangle$ fixes the set $\left\{x_{4}, x_{0}\right\}$ pointwise. Clearly $U_{6}$ and $U_{2}$ are conjugate in $H$. Also, it is clear that, given $u_{2} \in U_{2}^{\times}$, there exists $u_{6} \in U_{6}$ such that $U_{6}^{u_{2} u_{6}}=U_{2}$. Hence if $\left(U_{2}\right)_{\Omega} \cap\left(U_{2}^{\prime}\right)_{\Omega}$ is nontrivial, then we may take $u_{2} \in U_{2}^{\times}$such that its action on $\Omega$ coincides with an element $u_{2}^{\prime}$ of $U_{2}^{\prime \times}$. We thus obtain $\left(U_{2}\right)_{\Omega}=\left(U_{6}^{u_{2} u_{6}}\right)_{\Omega}=\left(U_{6}^{u_{2}^{\prime} u_{6}}\right)_{\Omega}=\left(U_{2}^{\prime}\right)_{\Omega}$.

So we may assume that $\left(U_{2}\right)_{\Omega}$ and $\left(U_{2}^{\prime}\right)_{\Omega}$ only share the identity (hence $|\Omega|>4$ ). This immediately implies that $\left(U_{2}\right)_{\Omega}$ centralizes $\left(U_{2}^{\prime}\right)_{\Omega}$. Since both $U_{2}$ and $U_{2}^{\prime}$ act regularly on $\Omega \backslash\left\{X_{1}\right\}$, we obtain $\left(U_{2}^{\prime}\right)_{\Omega}=\left(U_{2}\right)_{\Omega}^{\text {opp }}$.

Consider any line $\widetilde{X}_{1}$ incident with $x_{0}$, but different from $X_{1}$ and $X_{7}$, and define $\widetilde{x}_{2}$ and $\widetilde{X}_{3}$ as $\widetilde{X}_{1} I \widetilde{x}_{2} I \widetilde{X}_{3} I x_{4}$. Put $\widetilde{U}_{2}=G^{\widetilde{X}_{1}, \widetilde{x}_{2}, \widetilde{X}_{3}}$. Let $u_{6} \in U_{6}^{\times}$map $X_{1}$ to $\widetilde{X}_{1}$. We see that $\left(\widetilde{U}_{2}\right)_{\Omega}$ is conjugate to $\left(U_{2}\right)_{\Omega}$ in $\left\langle\left(U_{2}\right)_{\Omega},\left(U_{6}\right)_{\Omega}\right\rangle$ (via the action of $u_{6}$, but also via any element of $\left\langle\left(U_{2}\right)_{\Omega},\left(U_{6}\right)_{\Omega}\right\rangle$ mapping $X_{1}$ onto $\left.\widetilde{X}_{1}\right)$. Similarly, $\left(U_{2}\right)_{\Omega}$ is the conjugate of $\left(U_{6}\right)_{\Omega}$ in $\left\langle\left(\widetilde{U}_{2}\right)_{\Omega},\left(U_{6}\right)_{\Omega}\right\rangle$ by each element of the latter mapping $X_{7}$ onto $\widetilde{X}_{1}$. Now define

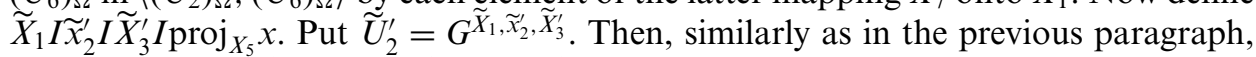
either $\left(\widetilde{U}_{2}^{\prime}\right)_{\Omega}=\left(\widetilde{U}_{2}\right)_{\Omega}$, or $\left(\widetilde{U}_{2}^{\prime}\right)_{\Omega}=\left(\widetilde{U}_{2}\right)_{\Omega}^{\text {opp }}$. In the former case we see, similarly as above, that $\left(U_{2}\right)_{\Omega}^{\text {opp }}$ is conjugate to $\left(U_{6}\right)_{\Omega}$ in $\left\langle\left(\widetilde{U}_{2}^{\prime}\right)_{\Omega},\left(U_{6}\right)_{\Omega}\right\rangle=\left\langle\left(\widetilde{U}_{2}\right)_{\Omega},\left(U_{6}\right)_{\Omega}\right\rangle$, implying by the observation above that $\left(U_{2}\right)_{\Omega}$ and $\left(U_{2}\right)_{\Omega}^{\text {opp }}$ are conjugate by any element of $\left\langle\left(\widetilde{U}_{2}\right)_{\Omega},\left(U_{6}\right)_{\Omega}\right\rangle$ fixing $X_{1}$, clearly a contradiction (as we can choose the identity!). Hence $\left(\widetilde{U}_{2}^{\prime}\right)_{\Omega}=\left(\widetilde{U}_{2}\right)_{\Omega}^{\text {opp }}$. This implies that $\left(U_{6}\right)_{\Omega}$ belongs to $\left\langle\left(U_{2}\right)_{\Omega}^{\text {opp }},\left(\widetilde{U}_{2}\right)_{\Omega}^{\text {opp }}\right\rangle$ and is in fact conjugate to $\left(U_{2}\right)_{\Omega}^{\text {opp }}$ by any element of $\left\langle\left(U_{2}\right)_{\Omega}^{\text {opp }},\left(\widetilde{U}_{2}\right)_{\Omega}^{\text {opp }}\right\rangle$ mapping $X_{1}$ onto $X_{7}$.

Now we know that there are at least five lines incident with $x_{0}$, so we may consider a line $\bar{X}_{7}$ incident with $x_{0}$, not belonging to $\left\{X_{1}, X_{7}, \widetilde{X}_{1}\right\}$. Define $\bar{X}_{7} I \bar{x}_{6} I \bar{X}_{5} I x_{4}$. We denote $\bar{U}_{6}:=G^{\bar{X}_{7}, \bar{x}_{6}, \bar{X}_{5}}$. We interchange the roles of $X_{7}$ and $\bar{X}_{7}$, and at the same time of $Y$ and $\bar{Y}:=\operatorname{proj}_{x} \operatorname{proj}_{x_{4}} \bar{X}_{7}$. We obtain, as in the previous paragraph, and using the fact that $\left(U_{2}^{\prime}\right)_{\Omega}$ does not depend on $Y$, but only on $x$, that $\left(\bar{U}_{6}\right)_{\Omega}$ belongs to $\left\langle\left(U_{2}\right)_{\Omega}^{\text {opp }},\left(\widetilde{U}_{2}\right)_{\Omega}^{\text {opp }}\right\rangle$ and is in fact conjugate to $\left(U_{2}\right)_{\Omega}^{\text {opp }}$ by any element of $\left\langle\left(U_{2}\right)_{\Omega}^{\text {opp }},\left(\widetilde{U}_{2}\right)_{\Omega}^{\text {opp }}\right\rangle$ mapping $X_{1}$ onto $\bar{X}_{7}$. It is now clear that, combining this with the conclusion of the previous paragraph, the group $\left\langle\left(U_{6}\right)_{\Omega},\left(\bar{U}_{6}\right)_{\Omega}\right\rangle$ contains $\left(U_{2}\right)_{\Omega}^{\text {opp }}$, conjugate to $\left(U_{6}\right)_{\Omega}$ by any element of $\left\langle\left(U_{6}\right)_{\Omega},\left(\bar{U}_{6}\right)_{\Omega}\right\rangle$ mapping $X_{7}$ to $X_{1}$. But of course $U_{2}$ is conjugate to $U_{6}$ by a (unique) element of $\bar{U}_{6}$, contradicting the fact that $\left(U_{2}\right)_{\Omega} \neq\left(U_{2}\right)_{\Omega}^{\mathrm{opp}}$.

The assertion is proved.

\section{REFERENCES}

1. A. E. Brouwer, The complement of a geometric hyperplane in a generalized quadrangle is usually connected, Finite geometry and combinatorics, Edited by F. De Clerck et al., London Math. Soc. Lecture Note Series No. 191 (Cambridge University Press, 1993), 53-57.

2. F. Buekenhout and H. Van Maldeghem, Finite distance transitive generalized polygons, Geom. Dedicata 52 (1994), 41-51.

3. P. Cameron, Orbits of permutation groups on unordered sets. II. J. London Math. Soc. (2) 23 (1981), 249-264.

4. J. D. Dixon and B. Mortimer, Permutation groups (Springer-Verlag, 1996). $1-57$. 

191-239.

6. P. Fong and G. M. Seitz, Groups with a (B,N)-pair of rank 2, II, Invent. Math. 21 (1974),

7. K. Tent, Half Moufang implies Moufang for generalized quadrangles, J. Reine Angew. Math., to appear.

8. K. Tent and H. Van Maldeghem, BN-pairs with affine or projective lines, J. Reine Angew. Math. 544 (2002), 223-236.

9. J. A. Thas, The classification of all $(x, y)$-transitive generalized quadrangles, J. Combin. Theory Ser. A 42 (1986), 154-157.

10. J. A. Thas, S. E. Payne and H. Van Maldeghem, Half Moufang implies Moufang for finite generalized quadrangles, Invent. Math. 105 (1991), 153-156.

11. K. Thas and H. Van Maldeghem, Geometrical characterizations of some Chevalley groups of rank 2, submitted.

12. J. Tits, Sur la trialité et certains groupes qui s'en déduisent, Inst. Hautes Études Sci. Publ. Math. 2 (1959), 13-60.

13. J. Tits, Classification of buildings of spherical type and Moufang polygons: a survey, Coll. Intern. Teorie Combin. Acc. Naz. Lincei, Roma 1973, Atti dei convegni Lincei 17 (1976), 229-246.

14. J. Tits, Endliche Spiegelungsgruppen, die als Weylgruppen auftreten, Invent. Math. $\mathbf{4 3}$ (1977), 283-295.

15. J. Tits, Moufang polygons, I. Root data, Bull. Belg. Math. Soc. Simon Stevin 1 (1994), $455-468$.

16. J. Tits and R. Weiss, Moufang polygons (Springer-Verlag, 2002).

17. H. Van Maldeghem, Generalized polygons (Birkhauser Verlag, 1998).

18. H. Van Maldeghem, J. A. Thas and S. E. Payne, Desarguesian finite generalized quadrangles are classical or dual classical, Designs, Codes and Cryptography 1 (1992), 299305 . 